\title{
Selpercatinib: First Approval
}

\author{
Anthony Markham ${ }^{1}$ \\ Published online: 17 June 2020 \\ (c) Springer Nature 2020, corrected publication 2020
}

\begin{abstract}
Selpercatinib (RETEVMO ${ }^{\mathrm{TM}}$ ) is a receptor tyrosine kinase RET (rearranged during transfection) inhibitor being developed by Loxo Oncology for the treatment of cancers harbouring RET alterations. Based on results from the phase I/II LIBRETTO-001 trial, selpercatinib was recently approved by the US FDA for the treatment of $R E T$ fusion-positive non-small-cell lung cancer, $R E T$ fusion-positive thyroid cancer and RET-mutant medullary thyroid cancer. This article summarizes the milestones in the development of selpercatinib leading to this first approval.
\end{abstract}

\section{Selpercatinib (RETEVMO'm): Key Points}

A receptor tyrosine kinase RET (rearranged during transfection) inhibitor is being developed by Loxo Oncology for the treatment of various cancers harbouring RET alterations

Received its first approval on 8th May 2020 in the US

Approved for use in adult patients with metastatic RET fusion-positive NSCLC, adult and paediatric patients $\geq 12$ years of age with advanced or metastatic $R E T$-mutant medullary thyroid cancer who require systemic therapy and adult and paediatric patients $\geq 12$ years of age with advanced or metastatic RET fusion-positive thyroid cancer who require systemic therapy and who are radioactive iodine-refractory (if radioactive iodine is appropriate)

Enhanced material for this AdisInsight Report can be found at https://doi.org/10.6084/m9.figshare.12431804.

This profile has been extracted and modified from the AdisInsight database. AdisInsight tracks drug development worldwide through the entire development process, from discovery, through preclinical and clinical studies to market launch and beyond.

Anthony Markham

dru@adis.com

1 Springer Nature, Private Bag 65901, Mairangi Bay, Auckland 0754, New Zealand

\section{Introduction}

Selpercatinib (RETEVMO ${ }^{\mathrm{TM}}$ ) is a highly selective receptor tyrosine kinase RET (rearranged during transfection) inhibitor developed by Loxo Oncology (Loxo, a wholly owned subsidiary of Eli Lilly and Company (Lilly) [1]) for the treatment of various solid tumours including non-small cell lung cancer (NSCLC) and thyroid cancer. Abnormally activated RET can act as an oncogene in various cancers; $R E T$ fusions retaining the kinase domain are drivers of NSCLC and papillary thyroid cancer, and activating RET mutations are associated with different phenotypes of multiple endocrine neoplasia type 2 and sporadic medullary thyroid cancer (MTC) [2]. Selpercatinib was approved in the US in May 2020 for the treatment of adult patients with metastatic RET fusion-positive NSCLC, adult and paediatric patients $\geq 12$ years of age with advanced or metastatic $R E T$-mutant MTC who require systemic therapy and adult and paediatric patients $\geq 12$ years of age with advanced or metastatic RET fusion-positive thyroid cancer who require systemic therapy and who are radioactive iodine-refractory (if radioactive iodine is appropriate) [3]. The recommended dose of the drug is $120 \mathrm{mg}$ (in patients weighing $<50 \mathrm{~kg}$ ) or $160 \mathrm{mg}$ (in patients weighing $\geq 50 \mathrm{~kg}$ ) orally twice daily $(\approx 12 \mathrm{~h})$ until disease progression or the emergence of unacceptable toxicity [4].

\subsection{Company Agreements}

In July 2013 Loxo entered into a multi-year licence and collaboration agreement with Array Biopharma (recently acquired by Pfizer [5]). The agreement covered intellectual 


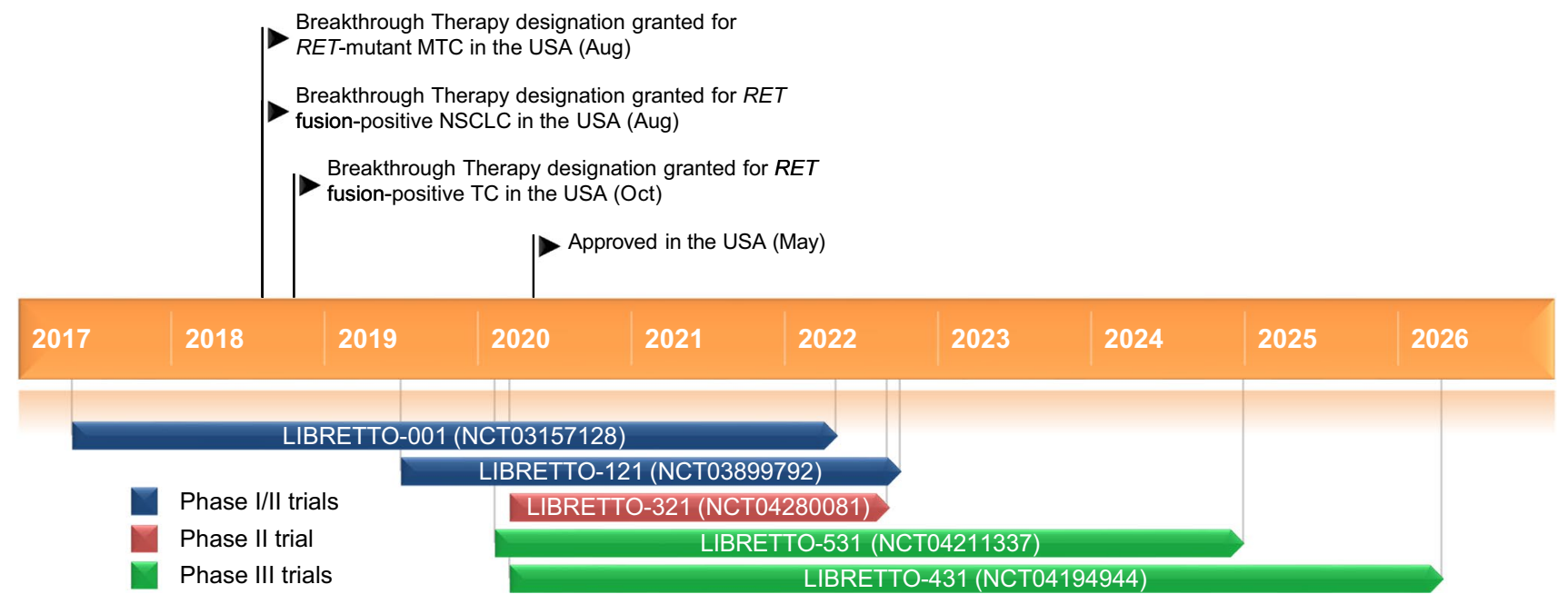

Key milestones in the development of selpercatinib leading to its approval for the treatment of for lung and thyroid cancers with RET gene mutations or fusions. CNS central nervous system, MTC medullary thyroid cancer, NSCLC non-small cell lung cancer

property related to selpercatinib, as well as the discovery and development of small-molecule drugs for mutually agreed oncology targets. Under the terms of the agreement, Loxo would fund preclinical research undertaken by Array and was responsible for target selection and clinical trials. Array would receive up to \$US434 million in milestone and royalty payments, as well as Loxo stock [6].

\section{Scientific Summary}

\subsection{Pharmacodynamics}

In vitro, selpercatinib potently inhibited proliferation of RET-mutant MTC cells (MZ-CRC-1 cell line [RETM918T]) $R E T$-mutant medullary thyroid carcinoma cells (TT cell line [RET C634W]) RET fusion-positive thyroid papillary carcinoma cells (TPC-1 cell line [CCDC6-RET]) and (NSCLC LC-2/Ad cell line [CCDC6-RET]) cells, but had 20- to 1700-fold less inhibitory activity against a panel of cell lines not containing RET alterations $(n=83)$. The drug was also associated with tumour regression in vivo in engineered and patient-derived $R E T$ fusion-positive and $R E T$-mutant murine models, including $R E T$ fusion-positive models harbouring the V804M acquired resistance gatekeeper mutation. In mice injected intracranially with CCDC6-RET fusion-positive PDX cell suspensions, orally administered selpercatinib was associated with significantly prolonged survival (median not reached) vs. vehicle (median 21 days) [7].

The effect of selpercatinib on the cardiac QTc interval has been studied in volunteers, with the largest mean increase in QTc predicted to be $10.6 \mathrm{~ms}$ at mean steady-state $\mathrm{C}_{\max }$ with selpercatinib $160 \mathrm{mg}$ administered twice daily [4].

\subsection{Pharmacokinetics}

In patients with cancer, selpercatinib $\mathrm{AUC}$ and $\mathrm{C}_{\max }$ at steady-state increased in a slightly greater than dose proportional manner at doses between 20 and $240 \mathrm{mg}$ twice daily (0.06-1.5 times the maximum recommended total daily dosage). Steady-state was reached after $\approx 7$ days with a 3.4 -fold median accumulation ratio at a dose of $160 \mathrm{mg}$ twice daily. Mean steady-state $\mathrm{C}_{\max }$ and $\mathrm{AUC}_{0-24 \mathrm{~h}}$ were $2980 \mathrm{ng} / \mathrm{mL}$ and $51600 \mathrm{ng} \cdot \mathrm{h} / \mathrm{mL}$, respectively, with a median $\mathrm{t}_{\max }$ of $2 \mathrm{~h}$. The mean absolute bioavailability of selpercatinib capsules was $73 \%$ in volunteers. Administration of selpercatinib with a high fat meal had no significant effect on selpercatinib AUC or $\mathrm{C}_{\max }$ in volunteers. The drug had an apparent clearance $(\mathrm{CL} / \mathrm{F})$ of $6 \mathrm{~L} / \mathrm{h}$ in patients and a $t_{1 / 2}$ of $32 \mathrm{~h}$ after oral administration to volunteers.

In volunteers, unchanged drug accounted for $86 \%$ of radioactivity after administration of a single oral $160 \mathrm{mg}$ radiolabelled dose of selpercatinib. Sixty nine and $24 \%$ of radioactivity was recovered in faeces and urine, respectively (14 and 12\% unchanged, respectively). The drug is primarily metabolised by cytochrome P450 (CYP) 3A4.<smiles>COc1ccc(CN2C3CCC2CN(c2ccc(-c4cc(OCC(C)(C)O)cn5ncc(C#N)c45)cn2)C3)cn1</smiles>

Chemical structure of selpercatinib 
Increasing body weight $(27-177 \mathrm{~kg})$ was associated with an increase in the apparent volume of distribution and clearance of selpercatinib. No clinically significant differences in the pharmacokinetics of selpercatinib were observed according to age (15-90 years), gender, or mild or moderate renal impairment, however, insufficient data are available to characterize the effect of severe renal impairment on the pharmacokinetic profile of the drug. The presence of mild, moderate and severe hepatic impairment increased the $\mathrm{AUC}_{0-\mathrm{INF}}$ of selpercatinib by $7 \%, 32 \%$, and $77 \%$, respectively, compared to subjects with normal hepatic function [4].

Coadministration of selpercatinib with multiple daily doses of omeprazole was associated with decreased selpercatinib $\mathrm{AUC}_{0-\mathrm{INF}}$ and $\mathrm{C}_{\max }$ when selpercatinib was administered during fasting but not when administered with food. Coadministration of selpercatinib with multiple daily doses of ranitidine had no clinically relevant effect on the pharmacokinetic profile of selpercatinib [4].

Following selpercatinib coadministration with multiple doses of the strong CYP3A inhibitor itraconazole, the $\mathrm{AUC}_{0-\mathrm{INF}}$ and $\mathrm{C}_{\max }$ of selpercatinib was increased by $133 \%$ and $30 \%$, respectively. Coadministration of selpercatinib with multiple doses of the moderate CYP3A inhibitors diltiazem, fluconazole, or verapamil is predicted to increase selpercatinib AUC (by $60-99 \%$ ) and $\mathrm{C}_{\max }$ (by 46-76\%) [4].

Coadministration of selpercatinib with multiple doses of the strong CYP3A inducer rifampin decreased the selpercatinib $\mathrm{AUC}_{0-\mathrm{INF}}$ and $\mathrm{C}_{\max }$ by $87 \%$ and $70 \%$, respectively; coadministration of selpercatinib with multiple doses of the moderate CYP3A inducers bosentan or efavirenz is predicted to decrease selpercatinib AUC and $\mathrm{C}_{\max }$ by $40-70 \%$ and $34-57 \%$, respectively, and coadministration of multiple doses of the weak CYP3A inducer modafinil is predicted to decrease the AUC and $\mathrm{C}_{\max }$ of selpercatinib by 33 and $26 \%$, respectively.

Coadministration of selpercatinib with the sensitive CYP2C8 substrate repaglinide increased the repaglinide $\mathrm{AUC}_{0-\mathrm{INF}}$ and $\mathrm{C}_{\max }$ by 188 and $91 \%$, respectively.

Coadministration of selpercatinib with the sensitive CYP3A substrate midazolam increased midazolam AUC 0-INF and $\mathrm{C}_{\max }$ by 54 and $39 \%$, respectively.

The pharmacokinetic profile of selpercatinib was not significantly affected when coadministered with a single dose of the P-glycoprotein inhibitor rifampin and no clinically significant differences in glucose levels were observed when the multidrug and toxin extrusion protein 1 (MATE1) substrate metformin was coadministered with selpercatinib [4].

\section{Features and properties of selpercatinib}

\begin{tabular}{|c|c|}
\hline Alternative names & LOXO-292, LY3527723 \\
\hline Class & Antineoplastics, aza compounds, ethers, nitriles, pyrazoles, pyridines \\
\hline Mechanism of Action & Receptor tyrosine kinase RET (rearranged during transfection) inhibitor \\
\hline Route of Administration & Oral \\
\hline Pharmacodynamics & Selective inhibitor of receptor tyrosine kinase RET \\
\hline Pharmacokinetics & Mean steady-state $\mathrm{C}_{\max } 2980 \mathrm{ng} / \mathrm{mL}, \mathrm{AUC}_{0-24 \mathrm{~h}} 51600 \mathrm{ng} \cdot \mathrm{h} / \mathrm{mL}$, median $\mathrm{t}_{\max } 2 \mathrm{~h}$ \\
\hline \multicolumn{2}{|l|}{ Adverse events } \\
\hline Most frequent & $\begin{array}{l}\text { Increased AST levels, increased AST levels, increased glucose levels, decreased albumin levels, decreased } \\
\text { leukocyte levels, decreased calcium levels, increased creatinine levels, dry mouth, diarrhoea, increased alka- } \\
\text { line phosphatase levels, hypertension, fatigue, decreased platelet levels, oedema, increased total cholesterol } \\
\text { levels, decreased sodium levels, rash, constipation, decreased magnesium levels, increased potassium levels, } \\
\text { increased bilirubin levels, headache, decreased glucose levels, nausea, abdominal pain, cough, prolonged QT } \\
\text { interval, dyspnoea, vomiting, haemorrhage }\end{array}$ \\
\hline Occasional & Hypersensitivity \\
\hline \multicolumn{2}{|l|}{ ATC codes } \\
\hline WHO ATC code & L01 (Antineoplastic Agents) \\
\hline EphMRA ATC code & L1 (Antineoplastics) \\
\hline Chemical name & $\begin{array}{l}\text { 6-(2-hydroxy-2-methylpropoxy)-4-(6-\{6-[(6-methoxypyridin-3-yl)methyl]-3,6-diazabicyclo[3.1.1]heptan- } \\
\text { 3-yl }\} \text { pyridin-3-yl)pyrazolo[1,5-a]pyridine-3-carbonitrile }\end{array}$ \\
\hline
\end{tabular}




\subsection{Therapeutic Trials}

\subsubsection{RET-Altered NSCLC}

Selpercatinib demonstrated marked antitumor activity in patients with RET fusion-positive NSCLC enrolled in the phase I/II LIBRETTO-001 trial (NCT03157128). In the initial phase I component of the study patients with advanced RET-altered solid tumours were treated with oral selpercatinib in 28-day cycles with the maximal tolerated dose and recommended dose for phase II trials established as $160 \mathrm{mg}$ twice daily [8]. Phase II enrolled patients to 1 of 6 cohorts based on tumour type, RET alteration and prior therapies [9]. At the data cutoff of 17 June 2019, 253 RET fusion-positive NSCLC patients had been treated. The primary analysis set for this report comprised the first 105 consecutively enrolled NSCLC patients who had received prior platinum-based chemotherapy, including 58 who had also received prior anti-programmed cell death protein 1 (PD-1)/PD-L1 therapy, most of whom were followed for $\geq 6$ months from first response; the data cutoff date for efficacy endpoint analyses was 16 December 2019. The overall response rate in the primary analysis set (primary endpoint) was $70 \%$ (73 of 105 patients) by investigator review and 64\% (67 of 105 patients) by independent review. The median duration of response was 20 months (median follow up of 15 months) by investigator review and 18 months (median follow-up of 12 months) by independent review [9]. The intracranial overall response rate was $81 \%$ by independent review (18 of 22 patients; 5 and 13 confirmed complete and partial responses, respectively) in patients with central nervous system target lesions at baseline [10]. The overall response rate in treatmentnaïve $R E T$ fusion-positive NSCLC patients $(\mathrm{n}=39)$ was $85 \%$ by independent review and $90 \%$ by investigator review; median duration of response was not reached [9].

\subsubsection{RET-Altered Thyroid Cancer}

Selpercatinib had marked antitumor activity in patients with RET-altered thyroid cancer enrolled in LIBRETTO-001. At the data cutoff of 17 June 2019, 226 patients with RETmutant MTC and 27 with RET fusion-positive thyroid cancer had been treated with selpercatinib. The primary analysis set for this report comprised the first 55 consecutively enrolled patients with $R E T$-mutant MTC previously treated with cabozantinib and/or vandetanib. The data cutoff date for efficacy endpoint analyses was 16 December 2019. The overall response rate in the primary analysis set (primary endpoint) was $62 \%$ (34 of 55 patients) by investigator review and 69\% (38 of 55 patients) by independent review. The median duration of response was not reached after a median 14.8 months' follow-up. The overall response rate in evaluable patients with RET fusion-positive thyroid cancer $(\mathrm{n}=19)$ was $58 \%$ by investigator review and $79 \%$ by independent review [11].

\subsubsection{RET-Altered Cancers in Paediatric Patients}

Preliminary data indicate selpercatinib is effective in paediatric patients with RET-altered cancers. Children ineligible to participate in clinical trials were treated with oral selpercatinib in continuous cycles, starting at a dose of $90 \mathrm{mg} / \mathrm{m}^{2}$ twice daily under regulatory agency-permitted, independent review board-approved single patient protocols. The first of two available reports detailed 4 cases aged between

Key clinical trials of selpercatinib (Loxo Oncology)

\begin{tabular}{|c|c|c|c|c|c|}
\hline $\operatorname{Drug}(\mathrm{s})$ & Indication & Phase & Status & Location(s) & Identifier \\
\hline Selpercatinib & $\begin{array}{l}\text { Advanced solid tumours, RET fusion-positive } \\
\text { solid tumours, and medullary thyroid cancer }\end{array}$ & I/II & Recruiting & Multinational & $\begin{array}{l}\text { NCT03157128, } \\
\text { LIBRETTO-001 }\end{array}$ \\
\hline Selpercatinib & $\begin{array}{l}\text { Advanced solid or primary CNS tumours in } \\
\text { paediatric patients }\end{array}$ & $\mathrm{I} / \mathrm{II}$ & Recruiting & US & $\begin{array}{l}\text { NCT03899792, } \\
\text { LIBRETTO-121 }\end{array}$ \\
\hline Selpercatinib & RET fusion-positive advanced NSCLC & II & Recruiting & US & NCT04268550 \\
\hline $\begin{array}{l}\text { Selpercatinib, cabozantinib, } \\
\text { vandetanib }\end{array}$ & RET-mutant medullary thyroid cancer & III & Recruiting & Multinational & $\begin{array}{l}\text { NCT04211337, } \\
\text { LIBRETTO-531 }\end{array}$ \\
\hline Selpercatinib & Solid tumours with RET activation & II & Recruiting & China & $\begin{array}{l}\text { NCT04280081, } \\
\text { LIBRETTO-321 }\end{array}$ \\
\hline $\begin{array}{l}\text { Selpercatinib, carboplatin, } \\
\text { cisplatin, pemetrexed, } \\
\text { pembrolizumab }\end{array}$ & $\begin{array}{l}\text { Advanced or metastatic RET fusion-positive } \\
\text { NSCLC }\end{array}$ & III & Recruiting & Multinational & $\begin{array}{l}\text { NCT04194944, } \\
\text { LIBRETTO-431 }\end{array}$ \\
\hline Selpercatinib & $\begin{array}{l}\text { Advanced solid tumours, lymphomas, or histio- } \\
\text { cytic disorders with activating RET gene altera- } \\
\text { tions, (a Paediatric MATCH Treatment Trial) }\end{array}$ & II & Pending & N/A & NCT04320888 \\
\hline Selpercatinib & $\begin{array}{l}\text { Solid tumours with evidence of activating RET } \\
\text { alteration (expanded access) }\end{array}$ & N/A & Available & Multinational & NCT03906331 \\
\hline
\end{tabular}


13 months and 8 years with tumours harbouring RET alterations; one from the Republic of Korea with papillary thyroid cancer, and one patient each with infantile myofibroma/ hemangiopericytoma, congenital mesoblastic nephroma/ infantile fibrosarcoma, or lipofibromatosis from the USA. As of 31 January 2019 the duration of treatment with selpercatinib ranged from 3 to 120 days with all patients continuing to receive selpercatinib. Partial responses-both ongoing, 1 confirmed, 1 pending confirmation-were observed in two patients evaluable for response [12]. The second report detailed five cases aged between 2 months and 15 years with tumours harbouring RET alterations, two with MTC, and one each with neuroblastoma, mesoblastic nephroma/ infantile fibrosarcoma or lipofibromatosis. As of 1 October 2019 four patients had partial response and one had stable disease [13].

\subsection{Adverse Events}

Adverse reactions (grade $1-4$ ) occurring in $\geq 25 \%$ of patients treated with selpercatinib in LIBRETTO-001 included dry mouth (39\%), diarrhoea (37\%), constipation (25\%), hypertension (35\%), fatigue (35\%), oedema (33\%), rash (27\%) [4]. Grades 3-4 adverse reactions occurring in $\geq 2 \%$ of patients were hypertension $(18.0 \%)$ and dyspnoea $(2.3 \%)$; grade 3 only adverse reactions were diarrhoea (3.4\%), fatigue $(2.0 \%)$, and prolonged QT interval (4\%).

Laboratory abnormalities ( $\geq 25 \%$ ) worsening from baseline in patients treated with selpercatinib in LIBRETTO-001 included increased AST levels (51\%), increased ALT levels (45\%), increased glucose levels (44\%), decreased albumin levels $(42 \%)$, decreased calcium levels (41\%), increased creatinine levels (37\%), increased alkaline phosphatase levels $(36 \%)$, increased total cholesterol levels $(31 \%)$, decreased sodium levels (27\%), decreased leukocyte levels (levels (43\%) and decreased platelet levels (33\%) [4]. Grade 3-4 laboratory abnormalities occurring in $\geq 2 \%$ of patients were increased AST levels (8\%), increased ALT levels (9\%), increased glucose levels $(2.2 \%)$, decreased calcium levels $(3.8 \%)$, increased alkaline phosphatase levels $(2.3 \%)$, increased bilirubin $(2.0 \%)$ and decreased platelets $(2.7 \%)$.

Serious adverse reactions were observed in $33 \%$ of patients treated with selpercatinib, most frequently pneumonia. Three percent of patients had fatal adverse reactions including sepsis, cardiac arrest and respiratory failure, each occurring in three patients. Five percent of patients required permanent discontinuation of selpercatinib therapy because of adverse reactions including increased ALT levels $(0.4 \%)$, sepsis $(0.4 \%)$, increased AST levels $(0.3 \%)$, drug hypersensitivity $(0.3 \%)$, fatigue $(0.3 \%)$, and thrombocytopenia $(0.3 \%)$. Dosage interruptions because of adverse reactions were required in $42 \%$ of patients treated with selpercatinib including increased ALT or AST levels, hypertension, diarrhoea, pyrexia, and QT prolongation; dosage reductions were required in $31 \%$ of patients because of adverse reactions including increased ALT or AST levels, QT prolongation and fatigue [4].

\subsection{Companion Diagnostic}

An FDA-approved test for the detection of RET gene fusions and RET gene mutations is not currently available [4]. Eli Lilly and Company has entered into an agreement with Thermo Fisher Scientific for the development of the Oncomine Dx Target Test, a qualitative in vitro diagnostic test that uses targeted high-throughput, parallel sequencing technology, as a companion diagnostic test for the selection of patients for whom selpercatinib would be indicated [14]. Additionally, Loxo has partnered with Illumina for next generation sequencing-based companion diagnostic development.

\subsection{Ongoing Clinical Trials}

The randomized phase III LIBRETTO-431 (NCT04194944) and LIBRETTO-531 (NCT04211337) trials are evaluating the efficacy of selpercatinib in patients with RET fusionpositive NSCLC and treatment-naïve RET-mutant MTC, respectively [15].

The phase II LIBRETTO-321 trial (NCT04280081) is underway in China evaluating the efficacy of selpercatinib in patients with advanced solid tumours including RET fusionpositive solid tumours, MTC and other tumours with RET activation.

LIBRETTO-121 (NCT03899792) is an ongoing multicentre phase I/II dose escalation multicentre trial in patients 6 months-21 years of age with advanced, RET-altered solid and CNS tumours [16].

\section{Current Status}

Selpercatinib received its first approval on 8th May 2020 in the US for the treatment of adult patients with metastatic RET fusion-positive NSCLC, adult and paediatric patients $\geq 12$ years of age with advanced or metastatic $R E T$-mutant MTC who require systemic therapy and adult and paediatric patients $\geq 12$ years of age with advanced or metastatic $R E T$ fusion-positive thyroid cancer who require systemic therapy and who are radioactive iodine-refractory (if radioactive iodine is appropriate). 


\section{Compliance with Ethical Standards}

Funding The preparation of this review was not supported by any external funding.

Conflict of interest During the peer review process the manufacturer of the agent under review was offered an opportunity to comment on the article. Changes resulting from any comments received were made by the authors on the basis of scientific completeness and accuracy. A. Markham is a contracted employee of Adis International Ltd/Springer Nature, is responsible for the article content and declares no relevant conflicts of interest.

Open Access This article is licensed under a Creative Commons Attribution-NonCommercial 4.0 International License, which permits any non-commercial use, sharing, adaptation, distribution and reproduction in any medium or format, as long as you give appropriate credit to the original author(s) and the source, provide a link to the Creative Commons licence, and indicate if changes were made. The images or other third party material in this article are included in the article's Creative Commons licence, unless indicated otherwise in a credit line to the material. If material is not included in the article's Creative Commons licence and your intended use is not permitted by statutory regulation or exceeds the permitted use, you will need to obtain permission directly from the copyright holder. To view a copy of this licence, visit http://creativecommons.org/licenses/by-nc/4.0/.

\section{References}

1. Eli Lilly, Loxo Oncology. Lilly completes acquisition of Loxo Oncology [media release]. 15 Feb 2019. http://www.lilly.com.

2. Subbiah V, Yang D, Velcheti V, et al. State-of-the-Art strategies for targeting RET-dependent cancers. J Clin Oncol. 2020;38(11):1209-21.

3. US Food \& Drug Administration. FDA approves selpercatinib for lung and thyroid cancers with RET gene mutations or fusions. Media Release. 2020.

4. Eli Lilly. RETEVMO ${ }^{\mathrm{TM}}$ (selpercatinib): US prescribing information. 2020. https://www.accessdata.fda.gov/drugsatfda_docs/label /2020/213246s000lbl.pdf. Accessed 2020.
5. Pfizer. Pfizer completes acquisition of Array Biopharma [media release]. $30 \mathrm{Jul}$ 2019. http://www.pfizer.com.

6. Loxo Oncology, Array BioPharma. Loxo Oncology and Array BioPharma announce license and collaboration agreement [media release]. $10 \mathrm{Jul}$ 2013. http://www.arraybiopharma.com.

7. Subbiah V, Velcheti V, Tuch BB, et al. Selective RET kinase inhibition for patients with RET-altered cancers. Ann Oncol. 2018;29(8):1869-76.

8. Drilon AE, Subbiah V, Oxnard GR, et al. A phase 1 study of LOXO-292, a potent and highly selective RET inhibitor, in patients with RET-altered cancers [abstract no. 102]. J Clin Oncol Conf. 2018;36(15 Suppl).

9. Goto K, Oxnard G, Tan D, et al. Selpercatinib (LOXO-292) in patients with RET-fusion + non-small cell lung cancer [abstract no. 3584 plus poster]. J Clin Oncol 2020;38(15 Suppl).

10. Subbiah V, Gainor JF, Oxnard G, et al. Intracranial activity of selpercatinib (LOXO-292) in RET fusion-positive non-small cell lung cancer (NSCLC) patients on the LIBRETTO-001 trial [abstract no. 9516 plus poster]. J Clin Oncol. 2020;38(15 Suppl).

11. Shah M, Sherman E, Robinson B, et al. Selpercatinib (LOXO-292) in patients with RET-mutant medullary thyroid cancer [abstract no. 3594 plus poster]. J Clin Oncol. 2020;38(15 Suppl).

12. Gerdemann U, Lee YA, Henry D, et al. First experience of LOXO292 in the management of pediatric patients with RET-altered cancers [abstract no. 10045]. J Clin Oncol. 2019;37(15 Suppl).

13. Ortiz MV, Gerdemann U, Govinda Raju S, et al. Activity of the highly specific RET inhibitor selpercatinib (LOXO-292) in pediatric patients with tumors harboring RET gene alterations. JCO Precis Oncol. 2020;4:341-7.

14. Thermo Fisher Scientific. Thermo fisher scientific signs agreement with Lilly Oncology for companion diagnostic to be used with RET inhibitor [media release]. 9 Sep 2019. http://www.thermofish er.com.

15. Eli Lilly. Lilly opens phase 3 clinical trial in RET-mutant medullary thyroid cancer [media release]. 30 Dec 2019. http://www.lilly .com.

16. DuBois SG, Albert CM, Mascarenhas L, et al. A phase I study of LOXO-292, a highly selective RET inhibitor, in pediatric patients with RET-altered cancers [abstract no. TPS10066]. J Clin Oncol. 2019;37(15 Suppl). 